Journal of Mathematics and Informatics

Vol. 7, 2017, 17-25

ISSN: 2349-0632 (P), 2349-0640 (online)

Published 16 March 2017

www.researchmathsci.org

DOI: http://dx.doi.org/10.22457/jmi.v7a3

Journal of

Mathematics and

Informatics

\title{
Research on Two-stage Supply Chain Coordination Model Based on Rebate and Penalty Contract
}

\author{
Ji-ming Zheng ${ }^{1}$ and Jiao-rui Jiang ${ }^{2}$
}

School of Science, Chongqing University of Posts and Telecommunications,

Chongqing - 400065, Chongqing, China.

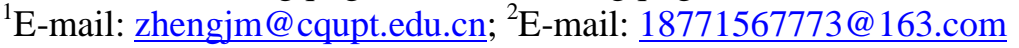

Received 2 March 2017; accepted 12 March 2017

\begin{abstract}
Based on the two-stage traditional supply chain model, which is composed of a manufacturer and a retailer, we introduce the online price parameter and online-offline sales volume proportion parameter, thus forming a two-stage supply chain conclude online and offline distribution channels under a stochastic demand environment. With the use of game theory, the interaction between the manufacturers and retailers can be observed. When contact parameters are satisfied, a coordination of the two-stage supply chain with online distribution channels can be realized through the rebate and penalty contract. Finally, we verify the availability of the contract by numerical calculating.
\end{abstract}

Keywords: Supply chain coordination; online channel; rebate and penalty contract; profit optimization

\section{AMS Mathematics Subject Classification (2010): 91B40}

\section{Introduction}

Supply chain is a chain of interests made up of different economic entities. The coordination of supply chain members is the foundation of supply chain stability. But, to pursuit the maximization of self-interest, the choice of the members in supply chain may conflict with the overall benefit of the supply chain, which lead to a certain degree of damage for the whole profits of supply chain. In order to coordinate the profit conflict between individual and whole in the supply chain, the supply chain contract has become an important means. Since Pasternack [1] early put forward the theory of supply chain contract, it had been developed rapidly in many directions. Bresnahan [2] studied the wholesale price contract from the perspective of economics in the case of deterministic demand. Yu etc. [3] proposed a wholesale price contract with anti-sudden events. Yang etc. [4] discussed the origin and development process of supply chain contract research, and established several basic mathematical models of typical supply chain contract. In the literature [5], researchers studied that, when the demand was associated with both effort factors and prices, the supply chain coordination can be achieved when introducing rebate and penalty strategy, and they also gave the model optimization method.

$\mathrm{Pu}$, etc. [6] found that the rebate and penalty contract could coordinate the supply chain effectively, making the upstream suppliers to supply on time. Liu, etc.[7] proposed resource-sharing contract theory based on rebate and penalty strategy service system . 


\section{Ji-ming Zheng and Jiao-rui Jiang}

$\mathrm{Xu}$, etc. [8] designed the difference repurchase contract based on the rebate and penalty strategy, and proved the contract could coordinate the supply chain model that existed customer returns. The literature [9-10] examined the coordination of the rebate and penalty contract on the closed-loop supply chain of emergency. Yang, etc. [11], studied that, when the market demand was uncertain, the coordination of supply chain can be achieved under the introduction of rebate and penalty contract in the two-stage return policy model.

All studies mentioned above mainly studied the coordination mechanism of the traditional two-stage supply chain. However, with the "Internet + " era, more and more manufacturers work on both online sales and offline sales. In order to adapt to the change of the market, it is necessary to increase the online sales channel in the traditional supply chain model. Although the researches on the traditional two-stage supply chain model are becoming more mature at present.

Based on the [4], this paper obtains a two-stage supply chain model with online sales channel, considering the introduction of the online sales proportion parameter and the online sales price parameter in the two-stage supply chain model. And it proved that the combination of traditional channel and online sales channel can enhance the overall profit of the two-stage traditional supply chain system. It demonstrated that using the contract of rebate and penalty can achieve the maximization of the benefits and the coordination of supply chain with online sales channel. Finally, a numerical example is given to show that the reasonable choice of contract parameters can realize the win-win of the members in supply chain without affecting the total profit of the supply chain system.

\section{Model description}

Considering one manufacturer and one retailer in the two-stage supply chain system, the manufacturer provides a single product to the retailer. The retailer's market demand is random and the retailer has only one chance to order before the selling season begins. According to the LF (Leader-Follower Game) game theory, the manufacturer is the leader and the retailer is the follower. The manufacturer will provide the retailer with a set of contract parameters, and the retailer determines the optimal product order quantity according to the contract parameters.

Assuming that the manufacturer is over-producing according to the order quantity provided by the retailer and is selling the excess part online, the manufacturer and the retailer are not only cooperative relationship, but also are certain competitive relationship. Assuming that the retail price of the unit product is $p$, and the online sales price is $p_{d}$. The ratio of the excess production produced by the manufacturer and the order quantity ordered by the retailer is $\alpha$, and the production cost of per unit product is $c$, and the wholesale price of the unit product provided by the manufacturer to the retailer is $\omega$, and the order quantity of the product is $Q$. Then we can construct a two-stage supply chain model with online sales channel. As shown in Fig. 1.

Before we build the model, we give the following basic assumptions for the twostage supply chain model: (i) market demand is random; (ii) the members of the supply chain is completely rational, that is, to maximize their own interests as the goal; (iii) the supply chain information is completely symmetrical; (iv) the members of the supply 


\section{Research on Two-stage Supply Chain Coordination Model Based on Rebate and Penalty Contract}

chain are risk-neutral; (v) online sales price will be greater than the wholesale price $\left(p_{d}>\omega\right)$, otherwise the retailer will order through the online sales channel.

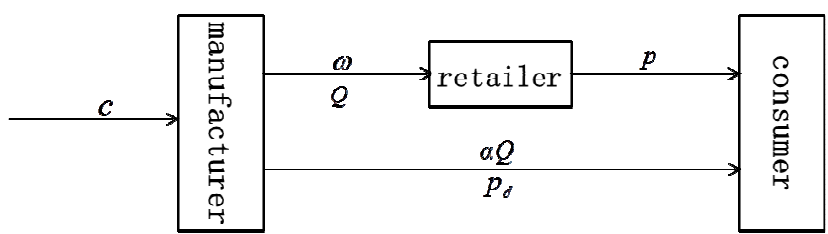

Figure 1: The two-stage supply chain with online sales channel

\section{Supply chain coordination}

\subsection{Decentralized decision}

When a two-stage supply chain model with an online sales channel is under decentralized decision, the manufacturer and the retailer trade through a wholesale price contract. The manufacturer provides a wholesale price to the retailer, and the retailer determines the order quantity before the start of sales season. At this time, the members aim at maximizing their own interests, and they will inevitably ignore the overall benefits of the supply chain system.

Assuming that the order quantity before the start of sales season is $Q$, and the excess production quantity formulated by the manufacturer is $\alpha Q$, and the wholesale price of the unit product is $\omega$, and the online sales price of the unit product is $p_{d}$, and the production cost of per unit product is $c$. For the manufacturer, the income from the retailer is $\omega Q$, the income from online sales is $p_{d} \alpha Q$, and the production cost to be paid is $c(1+\alpha) Q$, so the manufacturer's expected profit is

$$
\Pi_{m 1}=\omega Q+p_{d} \alpha Q-c(1+\alpha) Q .
$$

Assuming the retailer's expected sales volume is $S(Q)$, then $S(Q)=Q-\int_{0}^{Q} F(x) d x$, where $F(x)$ is the distribution function of the market demand $x$. After the retailer's sales season, assuming the expected value of the unsold products` quantity is $I(Q)$, and the unit product's loss of the retailer from excess order is $c_{e}$, and the unit product's loss of the retailer from out of stock is $c_{u}$. After the sales season, the residual value of the unit product that is not sold is $v$, and then the retailer's expected profit is

$$
\Pi_{R 1}=p S(Q)+v I(Q)-c_{e} I(Q)-c_{u} L(Q)-\omega Q .
$$

So, we can get

$$
\Pi_{R 1}=\left(p+c_{e}+c_{u}-v\right) S(Q)-\left(\omega+c_{e}-v\right) Q-c_{u} \mu .
$$

Since the expected profit of the supply chain system is the sum of the expected profits of the manufacturer and the retailer, the profit of the supply chain system is

$$
\begin{aligned}
\Pi_{T 1}= & \Pi_{m 1}+\Pi_{R 1} \\
& =p S(Q)+v I(Q)-c_{e} I(Q)-c_{u} L(Q)+p_{d} \alpha Q-c(1+\alpha) Q .
\end{aligned}
$$

Then, we can get

$$
\prod_{T 1}=\left(p+c_{e}+c_{u}-v\right) S(Q)-\left[c(1+\alpha)+c_{e}-v-p_{d} \alpha\right] Q-c_{u} \mu .
$$




\section{Ji-ming Zheng and Jiao-rui Jiang}

In particular, when the online-offline sales ratio $\alpha$ and the online sales price $p_{d}$ are equal to zero, the model is the traditional channel supply chain model under decentralized decision. Meanwhile, under the traditional channel model, the profit of the manufacturer $\Pi_{m 0}$, the profit of the retailer $\Pi_{R 0}$, and the profit of the supply chain system $\Pi_{T 0}$ can be obtained.

Therefore, under the decentralized decision-making, the retailer aims to maximize their own profit, and determine the order quantity of product, we can get the following proposition 1.

Proposition 1. [4] When the two-stage supply chain with online sales channel is under decentralized decision, the retailer's expected profit $\Pi_{R 1}$ is the concave function of the order quantity $Q$, and the optimal order quantity of the retailer is

$$
Q=F^{-1}\left(\frac{p+c_{u}-\omega}{p+c_{e}+c_{u}-v}\right) .
$$

where $F^{-1}(\bullet)$ is the inverse function of $F(x)$.

\subsection{Centralized decision}

Under centralized decision-making, manufacturer and retailer would form a strategic alliance to maximize system profit.

At this time, the income from the supply chain system through offline sales is $p S(Q)$, and the income earned through online sales is $p_{d} \alpha Q$. After the sales season, the income from the remaining products that are not sold is $v I(Q)$, and the cost of production that the supply chain system needs to pay out is $c(1+\alpha) Q$. Taking into account the loss caused by the shortage of products and the loss of the remaining products that were not sold after the sales season, the expected profit function $\Pi_{T 2}$ of the supply chain system can be obtained as follows:

$$
\Pi_{T 2}=p S(Q)+v I(Q)-c_{e} I(Q)-c_{u} L(Q)+p_{d} \alpha Q-c(1+\alpha) Q .
$$

Then, we can get

$$
\Pi_{T 2}=\left(p+c_{e}+c_{u}-v\right) S(Q)-\left[c(1+\alpha)+c_{e}-v-p_{d} \alpha\right] Q-c_{u} \mu .
$$

Under the centralized decision-making, manufacturers and retailers are equivalent to a whole. Then, the optimal order quantity of products can be given by the following proposition 2.

Proposition 2. When the two-stage supply chain with online sales channel is under centralized decision, the expected profit $\Pi_{T 2}$ of the supply chain system is the concave function of $Q$. Then, the optimal order quantity $Q_{T 2}$ of the supply chain system is

$$
Q_{T 2}=F^{-1}\left(\frac{p+c_{u}-\left[c-\left(p_{d}-c\right) \alpha\right]}{p+c_{e}+c_{u}-v}\right) .
$$

Proof: From (5), we can get

$$
\frac{d \prod_{T 2}}{d Q}=\left(p+c_{e}+c_{u}-v\right)(1-F(Q))-\left[c(1+\alpha) Q+c_{e}-v-p_{d} \alpha\right],
$$


Research on Two-stage Supply Chain Coordination Model Based on Rebate and Penalty Contract

$$
\frac{d^{2} \Pi_{T 2}}{d Q^{2}}=-\left(p+c_{e}+c_{u}-v\right) f(Q)<0 .
$$

Obviously, the expected profit $\Pi_{T 2}$ of the supply chain system is the strictly concave function of the order quantity $Q$. So the optimal order quantity $Q_{T 2}$ of the supply chain system should satisfy

$$
\frac{d \prod_{T 2}}{d Q}=0
$$

So, we can get

$$
F\left(Q_{T 2}\right)=\frac{p+c_{u}-\left[c-\left(p_{d}-c\right) \alpha\right]}{p+c_{e}+c_{u}-v} .
$$

Therefore, according to (7), under the centralized decision, it can be obtained that the optimal order quantity of the two-stage supply chain system with online sales channel is

$$
Q_{T 2}=F^{-1}\left(\frac{p+c_{u}-\left[c-\left(p_{d}-c\right) \alpha\right]}{p+c_{e}+c_{u}-v}\right) \text {. }
$$

Remark 1. Compared with the system profit of the two-stage supply chain under the centralized decision in [4], the online sales part is added, and the optimal order quantity is obtained when the system profit is maximized.

Remark 2. In the absence of any incentive measures, the two-stage supply chain with the online sales channel cannot achieve the maximal profits.

\subsection{The rebate and penalty contract}

By comparing (4) and (6), we find that the optimal order quantity of the retailer is less than the order quantity when the system profit is optimal. In order to achieve the optimal profit of the system, we need to take measures to encourage the retailer to increase product order quantity. Applying the rebate and penalty contract to the two-stage supply chain system with online sales channel, we assume that the sales target provided by the manufacturer to the retailer is $T$. After the sales season, if the number of products sold by the retailer exceeds $T$, for the excess part, the manufacturer will give the retailer rebate $\tau$ according to the unit product. If the number of products sold by the retailer do not exceeds $T$, the penalty is also $\tau$.

For the manufacturer's profit, applying the rebate and penalty contract to the supply chain system of decentralized decision-making, in addition to its income $\omega Q$ from retailers, and its income $p_{d} \alpha Q$ from online sales and the cost of production $c(1+\alpha) Q$, It is also required to deduct the reward $\tau[S(Q)-T]$ that the retailer has exceeded the sales target. When the expected sales volume $S(Q)$ is greater than the sales target $T$, it indicates that the manufacturer needs to reward the retailer. When the desired sales volume $S(Q)$ is less than the sales target $T$, it indicates that the manufacturer will punish the retailer. Thus, the manufacturer's expected profit under the contract is

$$
\Pi_{m}=\omega Q+p_{d} \alpha Q-\tau[S(Q)-T]-c(1+\alpha) Q .
$$

Similarly, the expected profit of the retailer under the contract is

$$
\Pi_{R}=p S(Q)+v I(Q)-c_{e} I(Q)-c_{u} L(Q)+\tau[S(Q)-T]-\omega Q .
$$


Ji-ming Zheng and Jiao-rui Jiang

Then, we can get

$$
\Pi_{R}=\left(p+c_{e}+c_{u}+\tau-v\right) S(Q)-\left(\omega+c_{e}-v\right) Q-c_{u} \mu .
$$

Since the expected profit of the supply chain system is the sum of the expected profits of the manufacturer and the retailer, the system profit of the supply chain under the contract is

$$
\begin{aligned}
\Pi_{T} & =\prod_{m}+\prod_{R} \\
& =p S(Q)+v I(Q)-c_{e} I(Q)-c_{u} L(Q)+p_{d} \alpha Q-c(1+\alpha) Q .
\end{aligned}
$$

Then, we can get

$$
\Pi_{T}=\left(p+c_{e}+c_{u}-v\right) S(Q)-\left[c(1+\alpha)+c_{e}-v-p_{d} \alpha\right] Q-c_{u} \mu .
$$

Proposition 3. In order to maximize the profits of the supply chain system and achieve the coordination of supply chain under the rebate and penalty contract, the contract parameters $\{\tau, T\}$ and related parameters need to satisfy the following conditions

$$
\left\{\begin{array}{l}
\tau>\frac{\left(p_{d}-c\right) \alpha\left(p+c_{e}+c_{u}-v\right)}{c+c \alpha+c_{e}-v-p_{d} \alpha} \\
c+c \alpha+c_{e}-v-p_{d} \alpha>0 \\
\frac{\prod_{T}+c_{u} \mu}{p+c_{e}+c_{u}-v}<T<\frac{1}{\tau\left(p+c_{e}+c_{u}-v\right)}\left[\left(p+c_{e}+c_{u}-v+\tau\right) \prod_{T}+\tau c_{u} \mu\right]
\end{array} .\right.
$$

Proof: From (9), we can get

$$
\begin{gathered}
\frac{d \prod_{R}}{d Q}=\left(p+c_{e}+c_{u}+\tau-v\right)(1-F(Q))-\left(\omega+c_{e}-v\right), \\
\frac{d^{2} \prod_{R}}{d Q^{2}}=-\left(p+c_{e}+c_{u}+\tau-v\right) f(Q)<0 .
\end{gathered}
$$

Obviously, $\Pi_{R}$ is the strictly concave function for the order quantity $Q$. At this time, the optimal order quantity of the retailer under the contract is satisfied

$$
Q_{R}=F^{-1}\left(\frac{p+c_{u}+\tau-\omega}{p+c_{e}+c_{u}+\tau-v}\right) .
$$

Similarly, from (10), the optimal order quantity $Q_{T}$ of the supply chain system under the contract is satisfied

Then, we can get

$$
F\left(Q_{T}\right)=\frac{p+c_{u}-\left[c-\left(p_{d}-c\right) \alpha\right]}{p+c_{e}+c_{u}-v} .
$$

$$
Q_{T}=F^{-1}\left(\frac{p+c_{u}-\left[c-\left(p_{d}-c\right) \alpha\right]}{p+c_{e}+c_{u}-v}\right) .
$$

At this time, to maximize the profit of the supply chain system, $Q_{T}$ should satisfy $Q_{T}=$ $=Q_{R}$. So, we can get

$$
\omega=\frac{\left(c+c \alpha-p_{d} \alpha\right)\left(p+c_{e}+c_{u}-v\right)+\tau\left(c+c \alpha+c_{e}-v-p_{d} \alpha\right)}{p+c_{e}+c_{u}-v} .
$$

Obviously, to achieve the supply chain coordination, the wholesale price $\omega$ should satisfy $\omega>c$, and from (13), we can get 
Research on Two-stage Supply Chain Coordination Model Based on Rebate and Penalty Contract

$$
\left\{\begin{array}{l}
\tau>\frac{\left(p_{d}-c\right) \alpha\left(p+c_{e}+c_{u}-v\right)}{c+c \alpha+c_{e}-v-p_{d} \alpha} \\
c+c \alpha+c_{e}-v-p_{d} \alpha>0
\end{array} .\right.
$$

Taking (14) into the retailer's profit function (9), there are

$$
\Pi_{R}=\frac{p+c_{e}+c_{u}-v+\tau}{p+c_{e}+c_{u}-v} \Pi_{T}+\frac{\tau}{p+c_{e}+c_{u}-v} c_{u} \mu-\tau T .
$$

Then, the manufacturer's profit is

$$
\Pi_{m}=\Pi_{T}-\Pi_{R}
$$

So, we can get

$$
\Pi_{m}=-\frac{\tau}{p+c_{e}+c_{u}-v} \Pi_{T}-\frac{\tau}{p+c_{e}+c_{u}-v} c_{u} \mu+\tau T .
$$

From (15), when $T<\frac{1}{\tau\left(p+c_{e}+c_{u}-v\right)}\left[\left(p+c_{e}+c_{u}-v+\tau\right) \prod_{T}+\tau c_{u} \mu\right], \Pi_{R}>0$. From (16), when $T>\frac{\Pi_{T}+c_{u} \mu}{p+c_{e}+c_{u}-v}, \quad \Pi_{m}>0$.Therefore, if the rebate and penalty contract can be used to achieve the coordination of supply chain, then sales target $T$ should satisfy

$$
\frac{\prod_{T}+c_{u} \mu}{p+c_{e}+c_{u}-v}<T<\frac{1}{\tau\left(p+c_{e}+c_{u}-v\right)}\left[\left(p+c_{e}+c_{u}-v+\tau\right) \prod_{T}+\tau c_{u} \mu\right] .
$$

Remark 3. (15) and (16) show that through the rational selection of parameters, you can achieve the system profit distribution among the supply chain members.

\section{Numerical examples}

Through the proposition 3, we can see that the profit of the supply chain system is affected by $p_{d}, \omega, \alpha$, and $\tau$ in different degrees. By changing the parameter $\tau, T$, the profit of supply chain system can be coordinated and the profit of the system can be distributed between the retailer and manufacturer. And it can ensure that the profit of each member in the supply chain under the contract is greater than that without the contract, to realize the coordination of the whole supply chain.

In order to facilitate the analysis and calculation, assuming that the market demand $x$ satisfies the uniform distribution on the interval $[a, b]$, then the probability density function of the market demand $x$ can be expressed as $f(x)=\frac{1}{b-a}$, the distribution function $F(x)=\frac{x-a}{b-a}$, then the inverse function of the distribution function is: $F^{-1}(x)=$ $a+x(b-a)$, expectation $\mu=\frac{a+b}{2}$, variance $\sigma^{2}=\frac{(b-a)^{2}}{12}$, then, $a$ and $b$ can be expressed as: $a=\mu-\sqrt{3} \sigma, b=\mu+\sqrt{3} \sigma$.

Assuming that the market demand satisfies the uniform distribution, in the numerical example, $p=35, c=5, c_{e}=2, c_{u}=2, v=3, \mu=100, \sigma=40, p_{d}=30, \omega=10$, 


\section{Ji-ming Zheng and Jiao-rui Jiang}

and $\alpha=0.08$. From (13), we can get $\tau=126$. It can be obtained that, through proposition 3 , if the rebate and penalty contract can achieve the coordination of the supply chain, the sales target $T$ needs to satisfy $94.2<T<119.5$. The Fig. 2 shows three cases' profit which include the supply chain of traditional channel, the two-stage supply chain with the online sales channel of the decentralized decision and the chain with the online sales channel of using rebate and penalty contract of manufacturers, retailers and supply chain systems change with the sales target $T$.

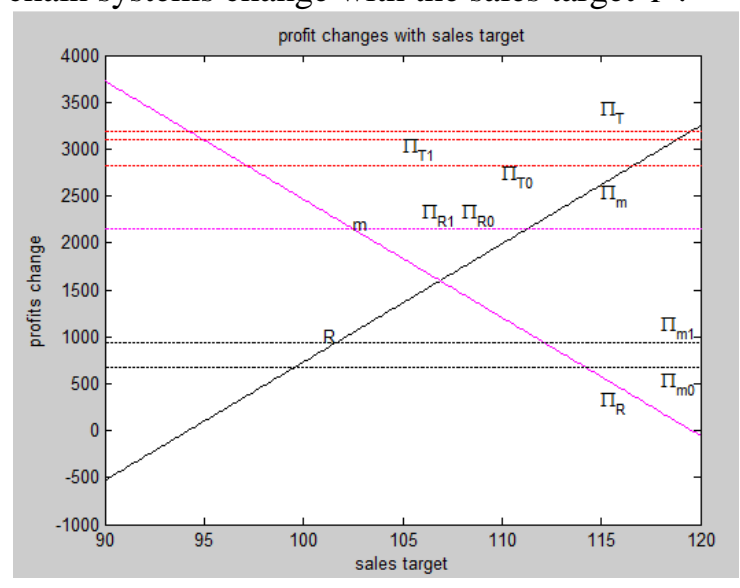

Figure 2: The impact of changes in sales targets on profits

Through Fig. 2, we can get that, the system profit under the contract of the two-stage supply chain with the online sales channel is always greater than the profit under the decentralized decision, and greater than the profit of traditional channel supply chain also. In the two-stage supply chain with online sales channel under the contract, the manufacturer can determine the sales target according to the market situation, to achieve the distribution of profits between the retailers and the manufacturers. We can get that the manufacturer's profits will increase with the increase of sales target, but the retailer's profit will decrease when the two-stage supply chain with online sales channel is under the contract. However, in order to achieve the coordination of the supply chain, it is required that the profits of the members under the contract should not be lower than the profits obtained under the decentralized decision. Therefore, the sales $\operatorname{target} T$ needs to satisfy $x_{R}<T<x_{m}$. At this time, $x_{R}$ satisfies $\Pi_{m}=\Pi_{m 1}$ and $x_{m}$ satisfies $\Pi_{R}=\Pi_{R 1}$. In this case, according to calculation result, $T$ can only be 102 to achieve the coordination of supply chain.

\section{Conclusions}

This paper proposed a two-stage supply chain model with online sales channel, and the system profit of the two-stage supply chain with online sales channel is better than that of the traditional channel supply chain under the decentralized decision-making. How to apply the rebate and penalty contract to realize the coordination of supply chain is also discussed, and proved that the contract parameter can maximize the profit of the supply chain in a certain range. Meanwhile, we got that on the basis of satisfying the profit maximization of the supply chain system, the profit of the manufacturer and the retailer 


\section{Research on Two-stage Supply Chain Coordination Model Based on Rebate and Penalty Contract}

can be improved at the same time by further restraining the contract parameter. In the future, we can consider the market demand under the fuzzy environment or information is not completely symmetrical and more complex market demand situation.

\section{REFERENCES}

1. B.A.Pasternack, Optimal pricing and return policies for perishable commodities, Marketing Science, 4 (1985) 166-176.

2. T.F.Bresnahan, Desler and manufacturer margins, RAND Journal of Economics, 16(2) (1985) 253-268.

3. H.Yu, J.Chen and G.Yu, Managing wholesale price contract in the supply chain under disruptions, Systems Engineering-Theory \& Practice, 26(8) (2006) 33-41.

4. D.Yang, Q.Guo and Y.He, et al., Review of supply chain contracts, Chinese Journal of Management, 3(1) (2006) 117-125.

5. Y.He, X.Zhao and L.Zhao, et al., Coordinating a supply chain with effort and price dependent stochastic demand, Applied Mathematical Modelling, 33(6) (2009) 27772790.

6. X.J.Pu, Q.Shi and L.Y.Ling, Penalty policy and incentive policy of JIT for multisupplier, Computer Integrated Manufacturing Systems, 2(11) (2007) 1876-1893.

7. B.C.Liu and J.Li, Resource-sharing shared contract model based on rebate and penalty strategy. Statistics \& Decision, 7 (2011) 62-64.

8. H.Xu, Q.L.Da and Y.Huang, Coordination mechanism of supply chain based on stochastic demand and customer returns, Journal of Southeast University, 42(1) (2012) 194-198.

9. Y.H.Qin and X.Y.Cao, The impact of emergencies on the closed-loop supply chain coordination under rebate and penalty contracts, Journal of Industrial Engineering and Engineering Management, 26(3) (2012) 96-101.

10. Y.H.Qin, Closed-loop supply chain coordination strategy with multi-factors disruptions simultaneously under emergency, Mathematics in Practice and Theory, (6) (2015)109-119.

11. C.Yang, S.Cheng and B.X.Zhang, Coordination and optimization of supply chain with consideration of uncertainty of the market demand, Mathematics in Practice and Theory, 17 (2016)129-135. 